Check for updates

Cite this: RSC Chem. Biol., 2022, 3,242

\section{A COVID-19 vaccine candidate composed of the SARS-CoV-2 RBD dimer and Neisseria meningitidis outer membrane vesicles $\dagger+$}

\author{
Darielys Santana-Mederos, (D) $\S^{a}$ Rocmira Perez-Nicado, (D) $\S^{a}$ Yanet Climent, (D) $\S^{a}$ \\ Laura Rodriguez, (D) $\S^{a}$ Belinda Sanchez Ramirez, $\S^{b}$ Sonia Perez-Rodriguez, ${ }^{c}$ \\ Meybi Rodriguez, ${ }^{a}$ Claudia Labrada, (D) a Tays Hernandez, ${ }^{\mathrm{b}}$ Marianniz Diaz, (D) ${ }^{\mathrm{b}}$ \\ Ivette Orosa, (D) ${ }^{b}$ Ubel Ramirez, ${ }^{a}$ Reynaldo Oliva, (D) ${ }^{a}$ Raine Garrido, (D) ${ }^{a}$ \\ Felix Cardoso, ${ }^{a}$ Mario Landys, ${ }^{a}$ Roselyn Martinez, ${ }^{a}$ Humberto Gonzalez, ${ }^{a}$ \\ Tamara Hernandez, ${ }^{a}$ Rolando Ochoa-Azze, ${ }^{a}$ Jose L. Perez, ${ }^{a}$ Juliet Enriquez, ${ }^{d}$ \\ Nibaldo Gonzalez, ${ }^{d}$ Yenicet Infante, ${ }^{d}$ Luis A. Espinosa, (D) ${ }^{\text {Y }}$ Yassel Ramos, \\ Luis Javier González, ${ }^{e}$ Carmen Valenzuela, ${ }^{\dagger}$ Ana Victoria Casadesus, ${ }^{b}$ \\ Briandy Fernandez, (DD ${ }^{b}$ Gertrudis Rojas, (DD ${ }^{b}$ Beatriz Pérez-Massón, (D) \\ Yaima Tundidor, (D) ${ }^{\mathrm{b}}$ Ernesto Bermudez, ${ }^{\mathrm{b}}$ Claudia A. Plasencia, ${ }^{\mathrm{b}}$ \\ Tammy Boggiano, (D) ${ }^{\mathrm{b}}$ Eduardo Ojito, (D) ${ }^{\mathrm{b}}$ Fabrizio Chiodo, (D) ag \\ Sonsire Fernandez, (D) a Françoise Paquet, (D) ${ }^{h}$ Cheng Fang, ${ }^{i}$ Guang-Wu Chen, $\S^{j}$ \\ Daniel G. Rivera, (D) $\S^{\star^{k}}$ Yury Valdes-Balbin, $\S^{\star^{a}}$ Dagmar Garcia-Rivera (D) $\S^{\star a}$ and \\ Vicente Verez Bencomo (D) $\S^{*^{a}}$
}

Received 19th October 2021, Accepted 1st December 2021

DOI: $10.1039 / \mathrm{d} 1 \mathrm{cb} 00200 \mathrm{~g}$

rsc.li/rsc-chembio
SARS-CoV-2 infection is mediated by the interaction of the spike glycoprotein trimer via its receptor-binding domain (RBD) with the host's cellular receptor. Vaccines seek to block this interaction by eliciting neutralizing antibodies, most of which are directed toward the RBD. Many protein subunit vaccines require powerful adjuvants to generate a potent antibody response. Here, we report on the use of a SARS-CoV-2 dimeric recombinant RBD combined with Neisseria meningitidis outer membrane vesicles (OMVs), adsorbed on alum, as a promising COVID-19 vaccine candidate. This formulation induces a potent and neutralizing immune response in laboratory animals, which is higher than that of the dimeric RBD alone adsorbed on alum. Sera of people vaccinated with this vaccine candidate, named Soberana01, show a high inhibition level of the RBD-ACE2 interaction using RBD mutants corresponding to SARS-CoV-2 variants of concern and wild-type expressed using the phage display technology. To our knowledge, this is the first time that the immunostimulation effect of $N$. meningitidis OMVs is evaluated in vaccine candidates against SARS-CoV-2.

\footnotetext{
${ }^{a}$ Finlay Vaccine Institute, 200 and 21 Street, Havana 11600,Cuba. E-mail: yvbalbin@finlay.edu.cu, dagarcia@finlay.edu.cu, vicente.verez@finlay.edu.cu

${ }^{b}$ Center of Molecular Immunology, P.O. Box 16040216 St., Havana, Cuba

${ }^{c}$ National Toxicology Center, Havana, 11500, Cuba

${ }^{d}$ National Civil Defense Research Laboratory, Mayabeque 32700, Cuba

${ }^{e}$ Center for Genetic Engineering and Biotechnology, Ave 31 e/158 y 190, Havana 10600, Cuba

${ }^{f}$ Institute of Cybernetics, Mathematics and Physics, Havana 10400, Cuba

${ }^{g}$ Department of Molecular Cell Biology and Immunology, Amsterdam UMC, Vrije Universiteit Amsterdam, Amsterdam, The Netherlands and Institute of Biomolecular Chemistry, National Research Council (CNR), Pozzuoli, Napoli, Italy

${ }^{h}$ Centre de Biophysique Moléculaire, CNRS UPR 4301, rue Charles Sadron, F-45071, Orléans Cedex 2, France

${ }^{i}$ Shanghai Fenglin Glycodrug Promotion Center, Shanghai 200032, China

${ }^{j}$ Chengdu Olisynn Biotech. Co. Ltd., and State Key Laboratory of Biotherapy and Cancer Center, West China Hospital, Sichuan University, Chengdu 610041, People's Republic of China

${ }^{k}$ Laboratory of Synthetic and Biomolecular Chemistry, Faculty of Chemistry, University of Havana, Zapata y G, Havana 1040o, Cuba. E-mail: dgr@fq.uh.cu

$\dagger$ Dedicated to the memory of Prof. José Luis García Cuevas

\$ Electronic supplementary information (ESI) available: Materials and methods, ESI-MS spectra, DLS and SE-HPLC chromatogram of the RBD dimer. See DOI: 10.1039/ d1 cb00200g

$\S$ These authors contributed equally to this work.
} 


\section{Introduction}

COVID-19 vaccines in use or clinical evaluation seek to induce protective immunity mainly by eliciting neutralizing antibodies $(\mathrm{NAbs})^{1-4}$ that block the interaction ${ }^{5-8}$ between the SARS-CoV-2 receptor-binding domain (RBD) of the spike $(S)$-glycoprotein and the host's cell surface receptor, the angiotensin-converting enzyme 2 (ACE2). Despite the rapid emergence of new platform technologies such as mRNA vaccines delivered by lipid nanoparticles and viral vector vaccines, ${ }^{1-4,9}$ recombinant subunit vaccines remain among the most effective ones to fight against SARS-CoV-2 due to their proven safety, clinical efficacy and ease of distribution. ${ }^{1-4,10,11}$ Two main types of COVID-19 subunit vaccines have advanced to clinical evaluation ${ }^{1-4}$ : those based on the full-length spike $(S)$-glycoprotein ectodomain ${ }^{11,12}$ and those using the RBD as the antigen. ${ }^{11,13}$ While the full $S$-glycoprotein is more immunogenic, the RBD is easier to produce and it also elicits a high-quality neutralizing response. ${ }^{13}$ The RBD can be obtained as a recombinant protein in a variety of expression systems, including insect cells, ${ }^{8,14}$ yeast $(P \text {. pastoris })^{15-17}$ and mammalian cells (CHO and HEK293). ${ }^{5,16,18}$

Different strategies have been implemented to increase the immunogenicity of RBD-based immunogens, including the use of potent adjuvants and multivalent antigen display in larger macromolecular constructs. ${ }^{13}$ The recombinant RBD has been conjugated to large protein carriers as tetanus toxoid ${ }^{19}$ and incorporated into self-assembled nanoparticles, ${ }^{13,20}$ leading to highly immunogenic multivalent constructs. Another approach has been the use of dimeric RBD immunogens, which have been produced in the form of fusion RBD-Fc chimeras ${ }^{21,22}$ or as an RBD dimer with a tandem-repeat single chain (sc-dimer) connecting the two RBD units. ${ }^{23}$ This RBD-sc-dimer - called vaccine ZF2001 - has completed phase I and II clinical trials ${ }^{24}$ with a three-dose schedule, proving to be safe and producing very high seroconversion of NAbs and a moderate cellular immune response.

Our endeavor on the use of the recombinant RBD as an antigen focuses on exploring different alternatives to improve its immunogenicity. RBD(Arg319-Phe541-(His) 6 ), which intentionally includes unpaired Cys538, ${ }^{19}$ was expressed in $\mathrm{CHO}$ cells as a mixture of the RBD monomer and dimer. The RBD monomer (RBD-m) was site-selectively conjugated to tetanus toxoid (TT) for producing an RBD-TT conjugate vaccine candidate, ${ }^{19}$ which recently completed phase III clinical evaluation under the name SOBERANA02 and received emergency approval in $\mathrm{Cuba}^{25}$ Here, we report on the development of a new vaccine candidate, SOBERANA01, containing the recombinant RBD dimer (RBD-d) and Neisseria meningitidis outer membrane vesicles (OMVs) adsorbed on alum. We recently communicated the successful use of the RBD-d adsorbed on alum as a booster vaccine for COVID-19 convalescents. ${ }^{26}$ Here, we describe the preparation and characterization of the RBD- $\mathrm{d}$ and compare the immunogenicity evaluation of formulations either containing or not $N$. meningitidis OMVs. The assessment of the RBD-ACE2 interaction inhibition of sera from vaccinated people using phage-displayed RBD mutants present in variants of concern (VOC) is also described, which sheds light on the effect of the adjuvant on the neutralizing response not only in mice but also in humans.

\section{Results and discussion}

\section{Vaccine design}

$N$. meningitidis OMVs, at $50 \mu \mathrm{g}$ per dose, are a component of antimeningococcal vaccine VAMENGOC-BC ${ }^{\circledR}$ that is produced on a large scale in Cuba, from which nearly 70 million doses have been applied in humans. ${ }^{27} N$. meningitidis OMVs are not only protective against meningitis, but also have well-known adjuvant properties associated with the stimulation of neutrophils, ${ }^{28}$ bone marrow-derived dendritic cells ${ }^{29}$ and macrophages, ${ }^{30}$ which are key players of the innate immune system recognizing pathogenassociated molecular patterns through toll-like receptors (TLR). Stimulation of dendritic cells and macrophages induces the production of two proinflammatory cytokines IL-1 $\beta$ and IL18, serving as a bridge with the adaptive immune system. ${ }^{31,32}$ These properties prompted us to explore the capacity of $N$. meningitidis OMVs for improving RBD-d immunogenicity initially in mice, and in case of satisfactory results, extend the study to the evaluation in humans.

\section{Preparation and characterization of the RBD dimer}

The formation of a stable disulfide-linked dimeric RBD has been observed for MERS-CoV RBD (Glu367-Tyr606) ${ }^{23}$ expressed in CHO cells and for SARS-CoV-2 RBD(Arg319-Phe541) expressed using the Bac-to-Bac baculovirus system. ${ }^{8}$ We focused on the production of the SARS-CoV-2 RBD in CHO cells with the sequence from residue 319 to 541 . This system proved to efficiently produce RBD-d bearing an intermolecular Cys538Cys538 disulfide bond (Fig. 1A), along with a monomeric RBD-Cys adduct due to the cysteinylation of Cys538 with free cysteine present in the culture medium. ${ }^{19}$ The RBD-d was separated from the monomer using size exclusion chromatography (SE-HPLC) in a Superdex 200 column (Fig. 1B). Consistent with the SE-HPLC results, dynamic light scattering (DLS) indicated the greater hydrodynamic diameter of the RBD dimer compared to the monomer (Fig. S1, ESI $\$$ ). The monomeric RBD-Cys adduct can be decysteinylated ${ }^{19}$ and eventually converted into RBD-d under oxidative conditions.

As shown in Fig. 1C, reduction of the dimer into a monomer was verified on SDS-polyacrylamide gel electrophoresis (SDSPAGE) by a band shift from $\sim 60 \mathrm{kDa}$ in non-reducing gel electrophoresis to $\sim 30 \mathrm{kDa}$ under reducing conditions. The ESI-MS spectrum of the PNGase-F deglycosylated RBD-d showed multiply-charged ions due to the heterogeneous nature of $O$-glycans (Fig. S3 in the ESI $\$$ ). The experimental molecular masses determined by ESI-MS confirm (a) the dimeric nature of this antigen and (b) the presence of four disulfide bonds at each RBD unit and the nature of $O$-glycosylations at Thr323/Ser325 residues (Table S1, ESI $\$$ ). In addition, after deglycosylation with PNGase-F in the presence of $N$-ethylmaleimide (NEM), the RBD dimer was digested with trypsin using an in-solution buffer-free digestion protocol, ${ }^{33}$ which allowed a full-sequence coverage 
A

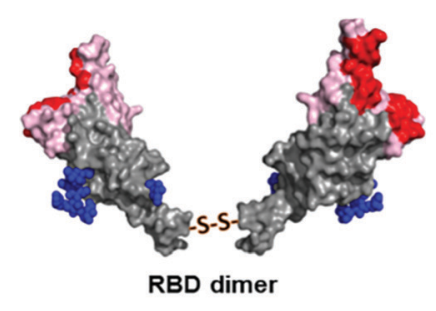

B

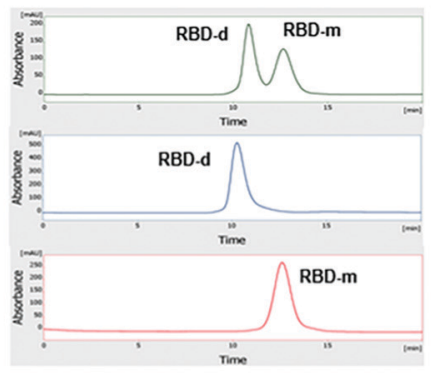

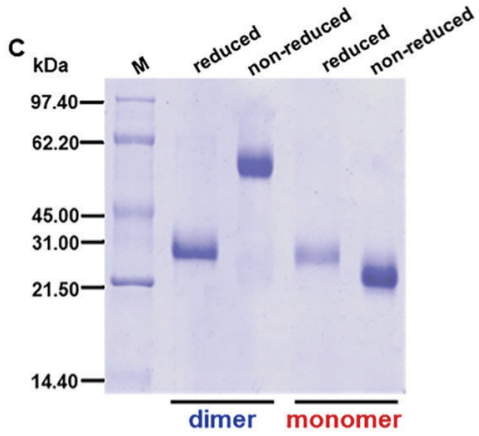

E
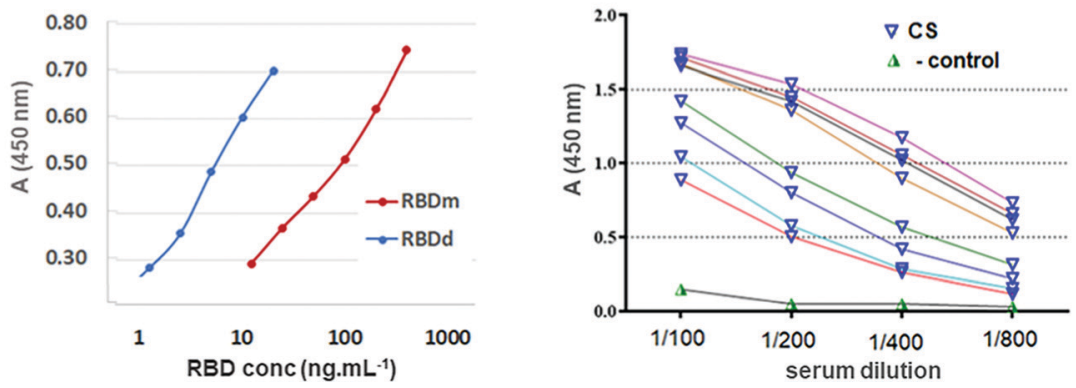

Fig. 1 Characterization of the recombinant RBD dimer. (A) Structure of the RBD dimer (red: amino acids contacting the ACE2 receptor upon binding, pink: RBM, blue: glycan chains, grey: RBD core). (B) SE-HPLC chromatograms of the RBD-d/RBD-m mixture and the purified antigens. (C) RBD- $d$ and RBD- $m$ in reducing and non-reducing SDS-PAGE. (D) Interaction of RBD- $d$ and RBD- $m$ with the ACE2 receptor expressed in Vero cells. (E) Recognition of RBD-d by eight convalescent sera from a Cuban panel.

including the identification of the intramolecular disulfide bonds. The confirmation of the disulfide bond linking the two RBD units at Cys538 came from the unequivocal identification of the $4+$ and $5+$ ions of the C-terminal peptide (Cys538-His547)-SS-(Cys538-His547). The assignment of all proteolytic peptides is listed in Table S2 in the ESI.+

To confirm the correct exposure of the receptor-binding motif (RBM) in the dimeric antigen, we analyzed by ELISA the interaction of the RBD-d with the ACE2 receptor either present on the surface of Vero cells (Fig. 1E) or with recombinant human ACE2 (hACE2-Fc, Fig. S4 in the ESI\$). Compared to the RBD-m, the dimeric antigen binds more efficiently to the ACE2 receptor. The RBD-d was also recognized by several convalescent sera from the Cuban panel (Fig. 1F), corroborating the good antigenic properties of this dimer.

\section{Immunogenicity in mice}

Previous immunogenicity studies on the RBD-sc-dimer proved that this immunogen induced a significantly higher anti-RBD IgG response compared to the RBD-m..$^{23}$ Accordingly, we also anticipated high immunogenicity for our disulfide-linked RBD-d, but at the same time, we sought to evaluate whether the potent innate-immunity stimulation with $N$. meningitidis OMVs can further improve the quality and the magnitude of the antiRBD immunity. Two different formulations were prepared: (a) RBD-d/OMV/alum $(10 \mu \mathrm{g} / 4 \mu \mathrm{g} / 250 \mu \mathrm{g})$ and (b) RBD-d/alum $(10 \mu \mathrm{g} / 250 \mu \mathrm{g})$. Balb/c mice were immunized following a threedose schedule (Fig. 2A). All animal procedures were performed in accordance with the Guidelines for Care and Use of Laboratory Animals of the Finlay Vaccine Institute and approved by its Animal Ethics Committee.
Compared to RBD-d/alum, the formulation containing RBD-d/OMV/alum induced a stronger IgG response already on day 14 after the first dose, and there was a significant $(p<0.05)$ increase in IgG titers at all times evaluated (Fig. 2B). In addition to the greater anti-RBD antibody response, the avidity index increased from 63.8 for RBD-d/alum to 80.7 for RBD-d/OMV/ alum (Fig. 2C). Similarly, both IgG1 (5.05 vs. 4.27) and IgG2a (3.89 vs. 2.15) titers were superior $(p<0.0001)$ for RBD-d/OMV/ alum than for the one lacking the bacterial OMVs (Fig. 2D). The increase of IgG2a and a higher IgG2a/IgG1 ratio (Fig. 2E) are consistent with the detected greater production of IFN- $\gamma$ (Fig. 2F). Altogether, these results indicate a more balanced Th1/Th2 $2^{34,35}$ response for RBD-d/OMV/alum, which is favorable for the prevention of COVID-19, as Th1 immune response helps viral clearing while Th2 cells are associated with poor prognosis. $^{36}$

RBD-specific memory B cells. There was a difference between RBD-d/OMV/alum and RBD-d/alum concerning the induction of RBD-specific memory $B$ cells. As shown in Fig. 3A, splenocytes collected on day 42 from mice immunized with these formulations were transferred intravenously to naive mice that were then boosted with a single dose of $10 \mu \mathrm{g}$ of RBD-d/ alum. There was a secondary RBD-specific IgG response for both formulations, but more intense for RBD-d/OMV/alum, with titers as high as $10^{2}$ to $10^{3}$ on day 7 after immunization.

Inhibition of the RBD-ACE2 interaction. To assess the functionality of the elicited anti-RBD antibodies, we measured the inhibition of the RBD-ACE2 receptor interaction in a molecular virus neutralization test $(\mathrm{mVNT})^{37}$ and a conventional virus neutralization test $\left(\mathrm{cVNT}_{50}\right)$ (Fig. 3). ${ }^{38} \mathrm{mVNT}$ is based on the antibody-mediated blockage of the RBD-hACE2 

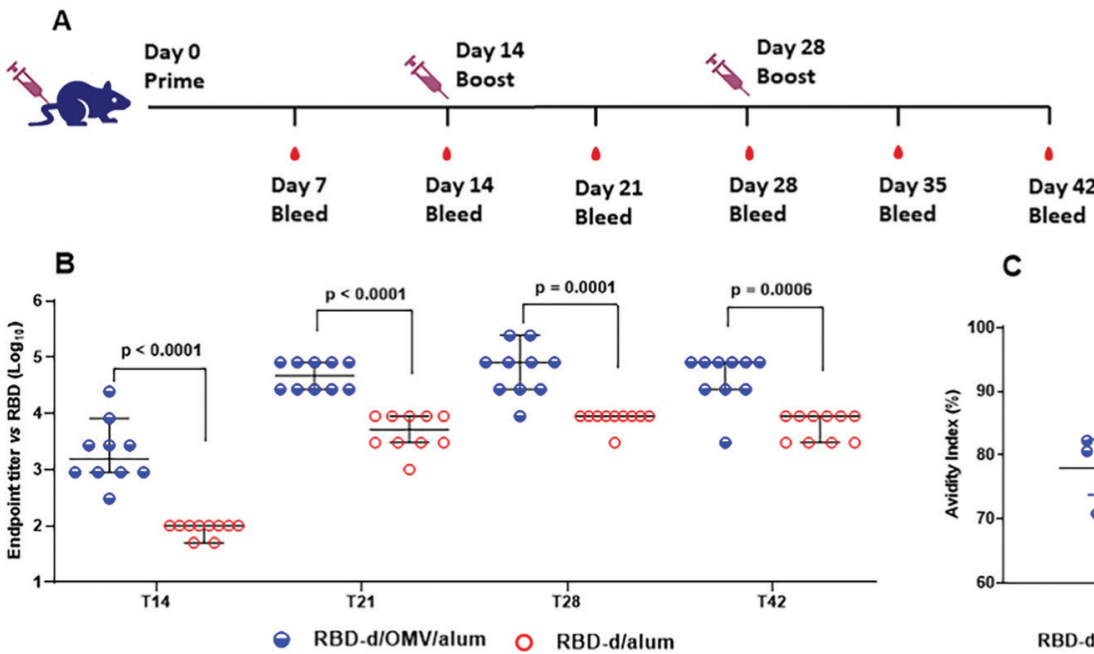

C
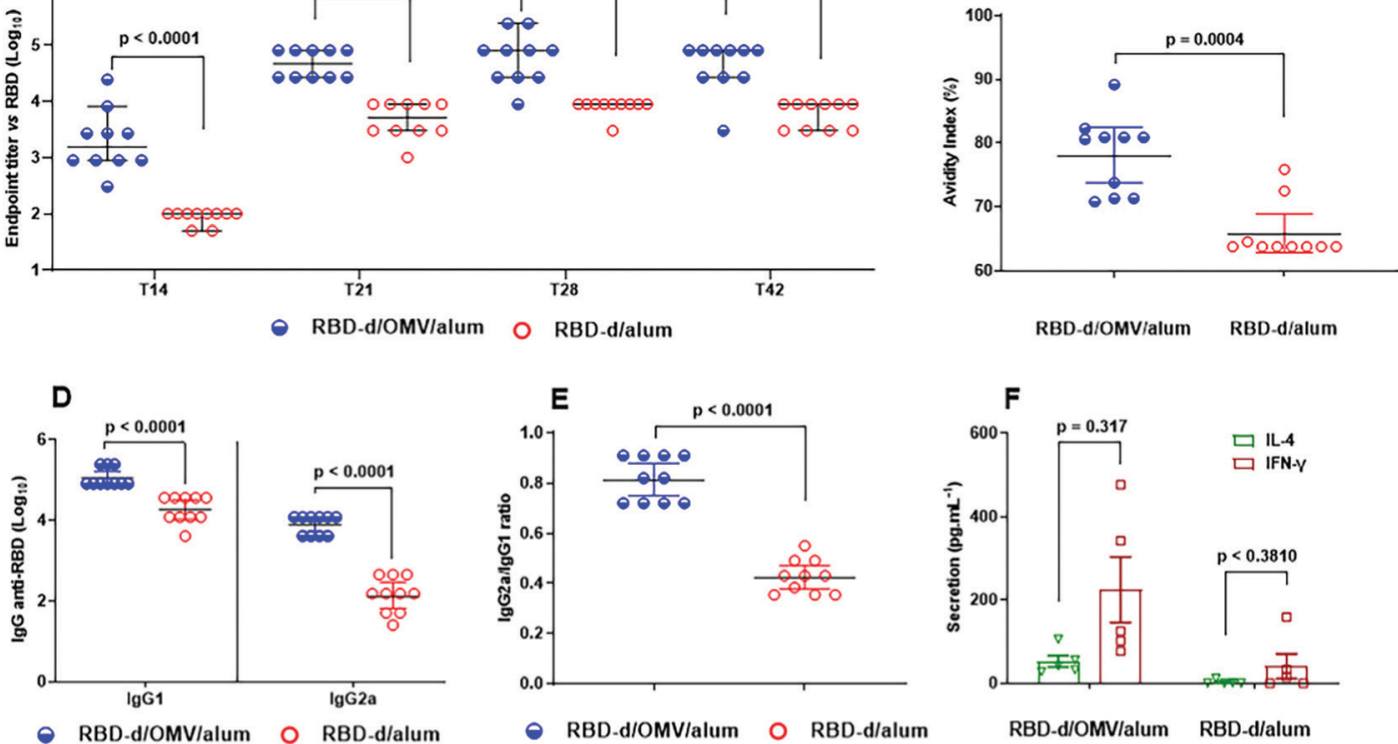

Fig. 2 Preclinical immunogenicity evaluation. Immunization of BALB/c mice with RBD-d/OMV/alum (blue) and RBD-d/alum (red). (A) Immunization protocol. (B) Anti-RBD IgG at days 14, 21, 28 and 42. (C) Avidity index of anti-RBD IgG elicited on day 42. (D) Anti-RBD IgG1 and IgG2a titers. (E) Anti-RBD IgG2a/lgG1 ratio. (F). Cytokine secretion (IL-4 and IFN- $\gamma$ ) after in vitro stimulation with RBD-d.

A

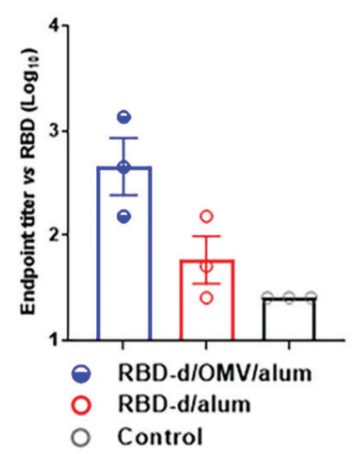

B



C

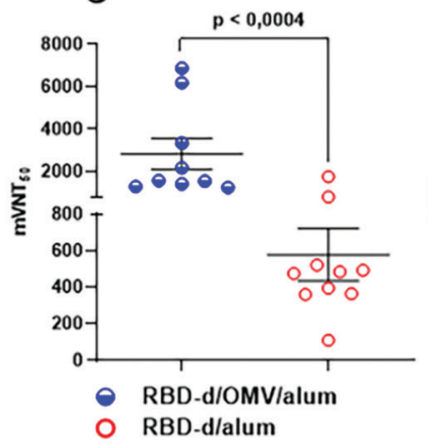

D

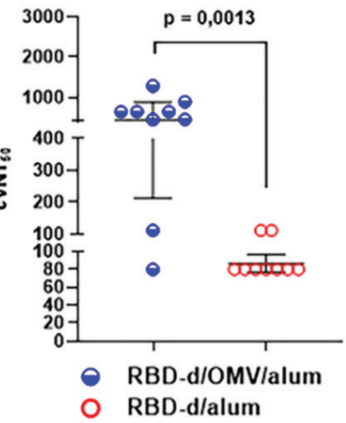

Fig. 3 Virus neutralization by anti-RBD antibodies induced by formulations RBD-d/OMV/alum and RBD-d/alum. (A) Classical passive transfer of splenocytes from BALB/c mice immunized with each vaccine formulation or placebo to naive recipient mice and stimulated with RBD-d/ alum (secondary response on day 7 after immunization). (B) Percentage of inhibition of the RBD-ACE2 interaction at $1 / 100$ serum dilution. (C) $\mathrm{mVNT}_{50}$ represents the serum dilution giving $50 \%$ inhibition of the RBD-ACE2 interaction. (D) $\mathrm{CVNT}_{50}$ measured as serum dilution giving $50 \%$ of virus neutralization.

interaction, and it is an in vitro surrogate of the virus neutralization test, detecting neutralizing antibodies in a short time. It uses the recombinant RBD mouse Fc (RBD-mFc) and the host-cell receptor hACE2-Fc as the coating antigen. Anti-RBD antibodies recognize the RBD-mFc, blocking its interaction with the ACE2 receptor. The RBD-mFc not inhibited by the antibodies can bind to the hACE2-Fc, which is recognized by a monoclonal antibody anti-gamma murine conjugated to alkaline phosphatase. This inhibition ELISA mimics the virus-host interaction at the molecular level. We initially evaluated the inhibition of the RBD-hACE2 interaction at 1/100 dilution of the sera of immunized mice. Mice immunized with the RBD-d/ 
OMV/alum formulation gave a higher $(p<0.0007)$ and more uniform inhibition than those immunized with RBD-d/alum (Fig. 3B). $\mathrm{mVNT}_{50}$, representing the serum dilution giving $50 \%$ inhibition of the ACE2-RBD interaction, also resulted in a significant difference $(p<0.0004)$ in the inhibition titers induced by RBD-d/OMV/alum compared to RBD-d/alum (Fig. 3C). The superior neutralizing response $(p=0.0013)$ elicited by RBD-d/OMV/alum was also confirmed in the $\mathrm{cVNT}_{50}$, which evaluates the inhibition of the interaction between the live SARS-CoV-2 virus and Vero E6 cells bearing ACE2 receptors. There was a higher level of neutralization for sera from most mice immunized with RBD-d/OMV/alum, while a lower NAb response was achieved after immunization with RBD-d/alum (Fig. 3D). In summary, $N$. meningitidis OMVs in the RBD-d formulation lead to a significant increase in the NAb response in mice compared to formulations containing only alum as the adjuvant. The presence of this bacterial adjuvant also makes the anti-RBD IgG response in mice comparable to that of the vaccine Soberana02, whose immunogen is an RBD-tetanus toxoid conjugate adjuvated in alum. ${ }^{19}$

\section{Human immunogenicity}

A phase I clinical trial was conducted to compare these and other vaccine candidates, in groups of 20 subjects following a three-dose schedule at intervals of 28 days. ${ }^{39}$ While the safety, reactogenicity and immunogenicity were reported, ${ }^{40}$ here we describe additional results which enable to further assess the effect of OMVs on the quality of the antibody response in humans. The analysis was performed with sera from trial participants vaccinated with RBD-d/ OMV/alum (50 $\mu \mathrm{g} / 20 \mu \mathrm{g} / 1250 \mu \mathrm{g})$ and RBD-d/alum (50 $\mu \mathrm{g} / 1250 \mu \mathrm{g})$.

Fig. 4A shows the anti-RBD IgG titer 28 days after the second $(T=56)$ and third doses $(T=84)$. The number of subjects with IgG concentration $>70 \mathrm{AU} / \mathrm{mL}$ increased after the third dose with respect to the second one: for RBD-d/OMV/alum from $13 / 20(65 \%)$ to $18 / 20$ (90\%) and for RBD-d/alum from 10/20 (50\%) to $13 / 20(65 \%){ }^{40}$ Interestingly, while the IgG response at day 28 after the second $(T=56)$ and third doses $(T=84)$ was rather similar, ${ }^{40}$ the quality of the IgG response was certainly higher for both formulations on day 28 after the third vaccination $(T=84)$, as determined by the inhibition of the RBD-ACE2 interaction. In $\mathrm{mVNT}_{50}$, the percentage of subjects attaining an $\mathrm{ID}_{50}>150$
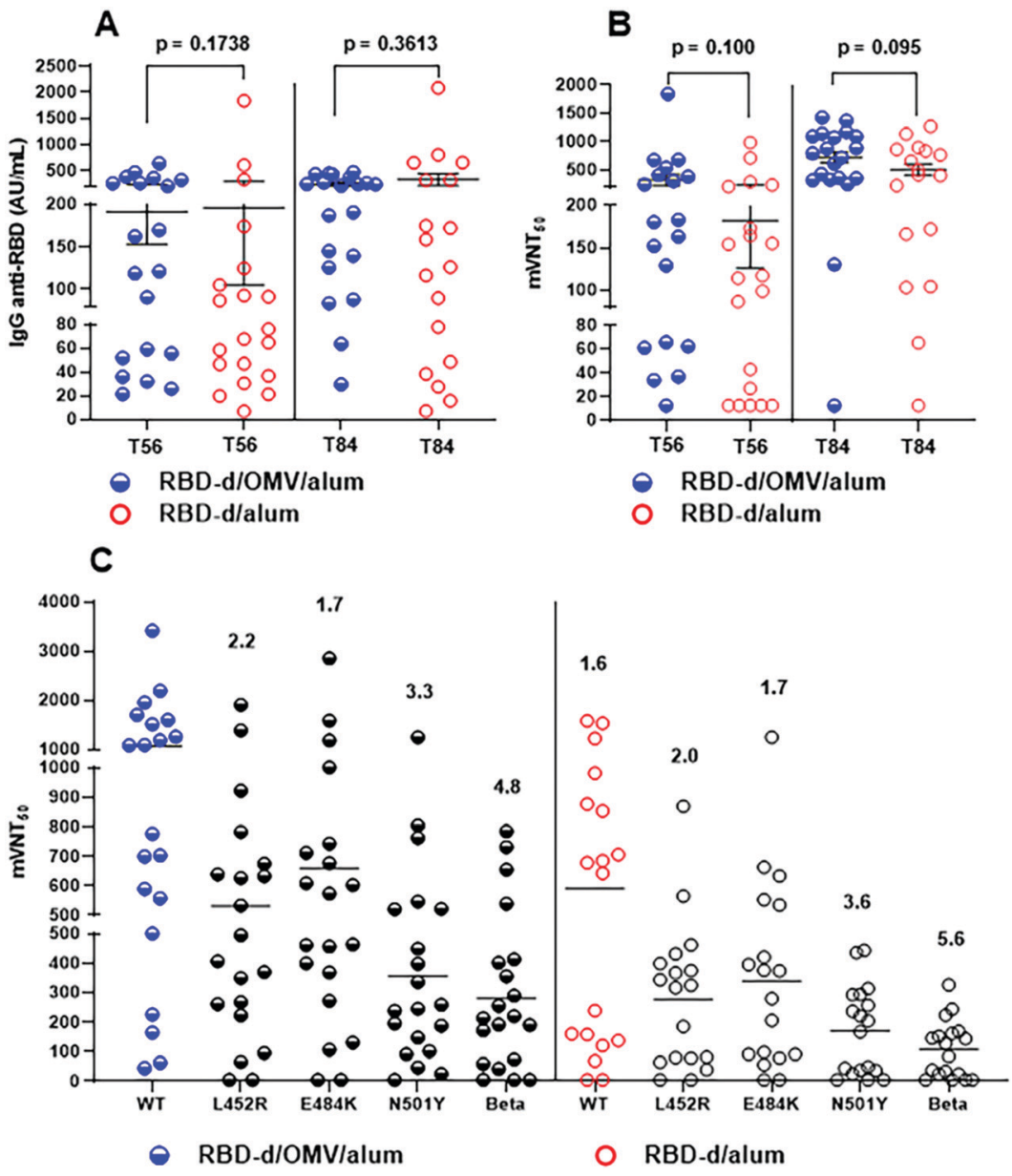

Fig. 4 Immunogenicity evaluation from a phase I clinical trial. ${ }^{39,40}$ Subjects immunized with RBD-d/OMV $(50 \mu g / 20 \mu g$, blue) vs. RBD-d (50 $\mu \mathrm{g}$, red) adsorbed in alum. (A) Anti-RBD IgG titers. (B) $\mathrm{mVNT}_{50}$ represents the serum dilution giving $50 \%$ inhibition of the RBD-ACE2 interaction. (C) $\mathrm{mVNT}_{50}$ comparing the inhibition of the interaction between the phage-displayed RBD mutants and the ACE2 receptor. Represented are the ratios of geometric mean titers (GMTs) between the RBD wild-type and the RBD mutants for each of the two vaccine formulations and the ratio of GMTs between the two formulations for the RBD wild-type. 
was $90 \%$ for the formulation RBD-d/OMV/alum and 55\% for that without OMVs (Fig. 4B). These results confirm the higher quality humoral response after vaccination with the OMV-containing formulation found in the preclinical evaluation.

Sera of vaccinated people were studied for their neutralizing effect against the RBD mutants of SARS-CoV-2 variants of concern (VOC), and compared with that against the RBD wild-type (Arg328Thr533). The single-mutant RBDs - L452R, E484K and N501Y and the triple-mutant one $\mathrm{K} 417 \mathrm{~N}, \mathrm{E} 484 \mathrm{~K}$ and N501Y, corresponding to the SARS-CoV-2 beta variant (B.1.351), were expressed using the phage display technology and used in a molecular virus neutralization test. As shown in Fig. 4C, the inhibition effect, determined as $\mathrm{mVNT}_{50}$, was higher for sera of people vaccinated with the OMV-containing formulation against the phage-displayed RBD wild-type and all mutants. For the RBD wild-type, the ratio of geometric mean titers (GMTs) between the subjects vaccinated with RBD-d/OMV/alum and with RBD-d/alum was 1.6. We also measured the GMT ratios between the RBD wild-type and the mutants for both formulations, showing a similar decrease in GMTs for phage-displayed RBD mutants L452R, E484K and N501Y compared to the wild-type. However, for the triplemutant corresponding to the beta variant, the GMTs declined 4.8-fold for sera of people vaccinated with RBD-d/OMV/alum, while the decrease was 5.6-fold for people vaccinated with RBD$\mathrm{d} /$ alum. Taken together, these results suggest that, in humans, the improvement in the quality of the antibody response due to the use of OMVs as the adjuvant leads to a better neutralizing effect not only against SARS-CoV-2 wild-type but also its variants.

\section{Conclusions}

We have demonstrated the substantial increase in immunogenicity and neutralizing IgG response of recombinant dimeric SARSCoV-2 RBD (319-541) when formulated together with $N$. meningitidis OMVs in alum. The RBD-d antigen bears an intermolecular S-S bond that connects the two RBD subunits, exposing well their receptor-binding motifs as demonstrated by its good antigenic properties. The innate-immunity stimulation provided by the OMV adjuvant contributes not only to the higher humoral response and better IgG functionality, but also to a more balanced Th1/Th2 response. In humans, the formulation containing the OMVs elicits a more potent neutralizing effect against RBD mutants present in VOCs, including the triple-mutant RBD of the beta variant. These results, together with those of phase I clinical trial, ${ }^{40}$ pave the way for phase II and III clinical trials for the RBD-d/OMV/alum formulation. They also demonstrate the advantages of using $N$. meningitidis OMVs not only in antibacterial vaccines, but also as adjuvants in antiviral protein subunit vaccines. This candidate, named Soberana01, adds a new asset to Cuba's research program on COVID-19 vaccines.

\section{Author contributions}

D. G.-R., Y. V.-B., and V. V.-B. conceived the idea and directed the research. D. S.-M., R. P.-N., Y. C., L. R., B. S. R., G.-W. C., and
D. G. R. proposed original ideas and conducted experimental work and data analysis. S. P.-R., M. R., C. L., T. H., M. D., I. O., U. R., R. O., R. G., F. C., M. L., R. M., H. G., T. H., R. O.-A., J. L. P., J. E., N. G., Y. I., L. A. E., Y. R., L. J. G., C. V., A. V. C., B. F., G. R., B. P.-M., Y. T., E. B., C. A. P., T. B., E. O., F. C., S. F., F. P., C. F., and G.-W. C. contributed to the experimental work and characterization. D. G. R., D. G.-R., and V. V.-B. wrote the article with contribution from all the authors.

\section{Conflicts of interest}

The authors declare no financial conflicts of interest. Some of the authors are co-inventors on a provisional SARS-CoV-2 vaccine patent $(\mathrm{Cu} 2020-57)$ based on these results.

\section{Acknowledgements}

We thank Rolando Pérez, Luis Herrera, Agustin Lage and Eduardo Martinez (BioCubaFarma) for advice and support to the project, and Lila Castellanos for scientific advice and corrections. We are grateful to Fondo de Ciencia e Innovación, CITMA-Cuba (Project FONCI 2020-20) and DAAD, Germany (Project GLACIER 57592717), for financial support.

\section{References}

1 F. Krammer, SARS-CoV-2 vaccines in development, Nature, 2020, 586, 516-527.

2 Y. Li, R. Tenchov, J. Smoot, C. Liu, S. Watkins and Q. Zhou, A Comprehensive Review of the Global Efforts on COVID-19 Vaccine Development, ACS Cent. Sci., 2021, 7, 512-533.

3 S. Kashte, A. Gulbake, S. F. El-Amin III and A. Gupta, COVID-19 vaccines: rapid development, implications, challenges and future prospects, Hum. Cell, 2021, 34, 711-733.

4 T. Thanh Le, Z. Andreadakis, A. Kumar, R. Gómez Román, S. Tollefsen, M. Saville and S. Mayhew, The COVID-19 Vaccine Development Landscape, Nat. Rev. Drug Discovery, 2020, 19, 305-306.

5 J. Shang, G. Ye, K. Shi, Y. Wan, C. Luo, H. Aihara, Q. Geng, A. Auerbach and F. Li, Structural basis of receptor recognition by SARS-CoV-2, Nature, 2020, 581, 221-224.

6 A. C. Walls, Y. J. Park, M. A. Tortorici, A. Wall, A. T. McGuire and D. Veesler, Structure, function, and antigenicity of the SARS-CoV-2 spike glycoprotein, Cell, 2020, 181, 281-292.

7 Y. Wan, J. Shang, R. Graham, R. S. Baric and F. Li, Receptor Recognition by the Novel Coronavirus from Wuhan: an Analysis Based on Decade-Long Structural Studies of SARS Coronavirus, J. Virol., 2020, 94, e00127-20.

8 J. Lan, J. Ge, J. Yu, S. Shan, H. Zhou, S. Fan, Q. Zhang, X. Shi, Q. Wang, L. Zhang and X. Wang, Structure of the SARS-CoV-2 spike receptor-binding domain bound to the ACE2 receptor, Nature, 2020, 581, 215-220.

9 M. D. Shin, S. Shukla, Y. H. Chung, V. Beiss, S. K. Chan, O. A. Ortega-Rivera, D. M. Wirth, A. Chen, M. Sack, J. K. Pokorski and N. F. Steinmetz, COVID-19 vaccine 
development and a potential nanomaterial path forward, Nat. Nanotechnol., 2020, 15, 646-655.

10 N. Wang, J. Shang, S. Jiang and L. Du, Subunit Vaccines Against Emerging Pathogenic Human Coronaviruses, Front. Microbiol., 2020, 11, 298.

11 J. Pollet, W. H. Chen and U. Strych, Recombinant protein vaccines, a proven approach against coronavirus pandemics, Adv. Drug Delivery Rev., 2021, 170, 71-82.

12 P. J. Hotez and M. E. Bottazzi, Whole Inactivated Virus and Protein-Based COVID-19 Vaccines, Annu. Rev. Med., 2022, 73, 24.1-24.10.

13 Y. Valdes-Balbin, D. Santana-Mederos, F. Paquet, S. Fernandez, Y. Climent, F. Chiodo, L. Rodríguez, B. Sanchez Ramirez, K. Leon, T. Hernandez, L. Castellanos-Serra, R. Garrido, G.-W. Chen, D. GarciaRivera, D. G. Rivera and V. Verez-Bencomo, Molecular Aspects Concerning the Use of the SARS-CoV-2 Receptor Binding Domain as a Target for Preventive Vaccines, ACS Cent. Sci., 2021, 7, 757-767.

14 J. Yang, W. Wang, Z. Chen, S. Lu, F. Yang, Z. Bi, L. Bao, F. Mo, X. Li, Y. Huang, W. Hong, Y. Yang, Y. Zhao, F. Ye, S. Lin, W. Deng, H. Chen, H. Lei, Z. Zhang, M. Luo, H. Gao, Y. Zheng, Y. Gong, X. Jiang, Y. Xu, Q. Lv, D. Li, M. Wang, F. Li, S. Wang, G. Wang, P. Yu, Y. Qu, L. Yang, H. Deng, A. Tong, J. Li, Z. Wang, J. Yang, G. Shen, Z. Zhao, Y. Li, J. Luo, H. Liu, W. Yu, M. Yang, J. Xu, J. Wang, H. Li, H. Wang, D. Kuang, P. Lin, Z. Hu, W. Guo, W. Cheng, Y. He, X. Song, C. Chen, Z. Xue, S. Yao, L. Chen, X. Ma, S. Chen, M. Gou, W. Huang, Y. Wang, C. Fan, Z. Tian, M. Shi, F.-S. Wang, L. Dai, M. Wu, G. Li, G. Wang, Y. Peng, Z. Qian, C. Huang, J. Y.-N. Lau, Z. Yang, Y. Wei, X. Cen, X. Peng, C. Qin, K. Zhang, G. Lu and X. Wei, A vaccine targeting the RBD of the $\mathrm{S}$ protein of SARS-CoV-2 induces protective immunity, Nature, 2020, 586, 572-577.

15 J. Pollet, W. H. Chen, L. Versteeg, B. Keegan, B. Zhan, J. Wei, Z. Liu, J. Lee, R. Kundu, R. Adhikari, C. Poveda, M. V. Mondragon, A. C. de Araujo Leao, J. A. Rivera, P. M. Gillespie, U. Strych, P. J. Hotez and M. E. Bottazzi, SARS-CoV-2 RBD219-N1C1: a yeast-expressed SARS-CoV-2 recombinant receptor-binding domain candidate vaccine stimulates virus neutralizing antibodies and t-cell immunity in mice, Hum. Vaccines Immunother., 2021, 1-11, DOI: 10.1080/21645515.2021.1901545.

16 Argentinian AntiCovid Consortium, Structural and functional comparison of SARS-CoV-2-spike receptor binding domain produced in Pichia pastoris and mammalian cells, Sci. Rep., 2020, 10, 21779.

17 M. Limonta-Fernández, G. Chinea-Santiago, A. M. MartínDunn, D. Gonzalez-Roche, M. Bequet-Romero, G. MarquezPerera, I. González-Moya, C. Canaan-Haden-Ayala, A. Cabrales-Rico, L. A. Espinosa-Rodríguez, Y. RamosGómez, I. Andujar-Martínez, L. J. González-López, M. Perez de la Iglesia, J. Zamora-Sanchez, O. Cruz-Sui, G. Lemos-Pérez, G. Cabrera-Herrera, J. Valdes-Hernández, E. Martinez-Diaz, E. Pimentel-Vazquez, M. Ayala-Avila and G. Guillén-Nieto, medRxiv, 2021, DOI: 10.1101/2021.06.29.21259605.
18 J. Zang, C. Gu, B. Zhou, C. Zhang, Y. Yang, S. Xu, L. Bai, R. Zhang, Q. Deng, Z. Yuan, H. Tang, D. Qu, D. Lavillette, Y. Xie and Z. Huang, Immunization with the receptor-binding domain of SARS-CoV-2 elicits antibodies cross-neutralizing SARS-CoV-2 and SARS-CoV without antibodydependent enhancement, Cell Discovery, 2020, 6, 61.

19 Y. Valdes-Balbin, D. Santana-Mederos, L. Quintero, S. Fernandez, L. Rodriguez, B. S. Ramirez, R. Perez, C. Acosta, Y. Méndez, M. G. Ricardo, T. Hernandez, G. Bergado, F. Pi, A. Valdes, U. Ramirez, R. Oliva, J.-P. Soubal, R. Garrido, F. Cardoso, M. Landys, H. Gonzalez, M. Farinas, J. Enriquez, E. Noa, A. Suarez, C. Fang, L. A. Espinosa, Y. Ramos, L. J. González, Y. Climent, G. Rojas, E. Relova, Y. Cabrera, S. L. Losada, T. Boggiano, E. Ojito, K. L. Monzon, F. Chiodo, F. Paquet, G.-W. Chen, D. G. Rivera, D. Garcia-Rivera and V. Verez Bencomo, SARSCoV-2 RBD-Tetanus toxoid conjugate vaccine induces a strong neutralizing immunity in preclinical studies, ACS Chem. Biol., 2021, 16, 1223-1233.

20 A. C. Walls, B. Fiala, A. Schafer, S. Wrenn, M. N. Pham, M. Murphy, L. V. Tse, L. Shehata, M. A. O’Connor, C. Chen, M. J. Navarro, M. C. Miranda, D. Pettie, R. Ravichandran, J. C. Kraft, C. Ogohara, A. Palser, S. Chalk, E. C. Lee, K. Guerriero, E. Kepl, C. M. Chow, C. Sydeman, E. A. Hodge, B. Brown, J. T. Fuller, K. H. Dinnon 3rd., L. E. Gralinski, S. R. Leist, K. L. Gully, T. B. Lewis, M. Guttman, H. Y. Chu, K. K. Lee, D. H. Fuller, R. S. Baric, P. Kellam, L. Carter, M. Pepper, T. P. Sheahan, D. Veesler and N. P. King, Elicitation of potent neutralizing antibody responses by designed protein nanoparticle vaccines for SARS-CoV-2, Cell, 2020, 183, 1367-1382e17.

21 Z. Liu, W. Xu, S. Xia, C. Gu, X. Wang, Q. Wang, J. Zhou, Y. Wu, X. Cai, D. Qu, T. Ying, Y. Xie, L. Lu, Z. Yuan and S. Jiang, RBD-Fc-based COVID-19 vaccine candidate induces highly potent SARS-CoV-2 neutralizing antibody response, Signal Transduction Targeted Ther., 2020, 5, 282.

22 S. Sun, L. He and Z. Zhao, et al., Recombinant vaccine containing an RBD-Fc fusion induced protection against SARS-CoV-2 in nonhuman primates and mice, Cell. Mol. Immunol., 2021, 18, 1070-1073.

23 L. Dai, T. Zheng, K. Xu, Y. Han, L. Xu, E. Huang, Y. An, Y. Cheng, S. Li, M. Liu, M. Yang, Y. Li, H. Cheng, Y. Yuan, W. Zhang, C. Ke, G. Wong, J. Qi, C. Qin, J. Yan and G. F. Gao, A universal design of betacoronavirus vaccines against COVID-19, MERS, and SARS, Cell, 2020, 182, 722-733e11.

24 S. Yang, Y. Li, L. Dai, J. Wang, P. He, C. Li, X. Fang, C. Wang, X. Zhao, E. Huang, C. Wu, Z. Zhong, F. Wang, X. Duan, S. Tian, L. Wu, Y. Liu, Y. Luo, Z. Chen, F. Li, J. Li, X. Yu, H. Ren, L. Liu, S. Meng, J. Yan, Z. Hu, L. Gao and G. F. Gao, Safety and immunogenicity of a recombinant tandemrepeat dimeric RBD-based protein subunit vaccine (ZF2001) against COVID-19 in adults: two randomised, double-blind, placebo-controlled, phase 1 and 2 trials, Lancet Infect. Dis., 2021, 21, 1107-1119.

25 International Clinical Trials Registry Platform. Identifier RPCEC00000354 Phase III clinical trial, multicenter, adaptive, 
parallel-group, randomized, placebo-controlled, double-blind study to evaluate the efficacy, safety and immunogenicity of vaccination against SARS-CoV-2 with 2 doses of FINLAY-FR-2 and a heterologous scheme with 2 doses of FINLAY-FR-2 and a booster dose with FINLAY-FR-1A (COVID-19). https://rpcec.sld. cu/en/trials/RPCEC00000354-En.

26 A. Chang-Monteagudo, R. Ochoa-Azze, Y. Climent-Ruiz, C. Macías-Abraham, L. Rodríguez-Noda, C. Valenzuela-Silva, B. Sánchez-Ramírez, R. Perez-Nicado, T. Hernández-García, I. Orosa-Vázquez, M. Díaz-Hernández, M. A. García-García, Y. Jerez-Barceló, Y. Triana-Marrero, L. Ruiz-Villegas, L. D. Rodríguez-Prieto, R. Puga-Gómez, P. P. Guerra-Chaviano, Y. Zúñiga-Rosales, B. Marcheco-Teruel, M. Rodríguez-Acosta, E. Noa-Romero, J. Enríquez-Puertas, D. Porto-González, O. Fernández-Medina, A. Valdés-Zayas, G.-W. Chen, L. Herrera-Martínez, Y. Valdés-Balbín, D. García-Rivera and V. Verez-Bencomo, A single dose of SARS-CoV-2 FINLAY-FR-1A vaccine enhances neutralization response in COVID-19 convalescents, with a very good safety profile: An open-label phase 1 clinical trial, Lancet Reg. Health Americas, 2021, 4, 100079.

27 R. F. Ochoa-Azze, L. García-Imía and V. Vérez-Bencomo, Effectiveness of a Serogroup B and C Meningococcal Vaccine Developed in Cuba, MEDICC Rev., 2018, 20, 22-29.

28 J. A. Lapinet, P. Scapini, F. Calzetti, O. Pérez and M. A. Cassatella, Gene Expression and Production of Tumor Necrosis Factor Alpha, Interleukin-1 $\beta$ (IL-1 $\beta$ ), IL-8, Macrophage Inflammatory Protein $1 \alpha$ (MIP-1 $\alpha$ ), MIP-1 $\beta$, and Gamma Interferon-Inducible Protein 10 by Human Neutrophils Stimulated with Group B Meningococcal Outer Membrane Vesicles, Infect. Immun., 2000, 68, 6917-6923.

29 C. Mesa, J. de León, K. Rigley and L. E. Fernández, Very small size proteoliposomes derived from Neisseria meningitidis: an effective adjuvant for Th1 induction and dendritic cell activation, Vaccine, 2004, 22, 3045-3052.

30 T. Rodríguez, O. Pérez, N. Menagerb, S. Ugrinovicb, G. Bracho and P. Mastroenibet, Interactions of proteoliposomes from serogroup B Neisseria meningitidis with bone marrow-derived dendritic cells and macrophages: adjuvant effects and antigen delivery, Vaccine, 2005, 23, 1312-1321.

31 T. Ito, J. M. Connette, S. L. Kunkel and A. Matsukawa, The linkage of Innate and adaptive immune responses during granulomatous development, Front. Immunol., 2013, DOI: 10.3389/fimmu.2013.00010.

32 C. A. Dinarello, D. Novick, S. Kim and G. Kaplanski, Interleukin-18 and interleukin-18 binding protein, Front. Immunol., 2013, DOI: 10.3389/fimmu.2013.00289.
33 L. H. Betancourt, L. A. Espinosa, Y. Ramos, M. BequetRomero, E. N. Rodríguez, A. Sánchez, G. Marko-Varga, L. J. González and V. Besada, Targeting hydrophilic regions of recombinant proteins by MS using in-solution buffer-free trypsin digestion, Eur. J. Mass Spectrom., 2020, 26, 230-237.

34 T. L. Stevens, A. Bossie, V. M. Sanders, R. Fernandez-Botran, R. L. Coffman, T. R. Mosmann and E. S. Vitetta, Regulation of antibody isotype secretion by subsets of antigen-specific helper T cells, Nature, 1988, 334, 255-258.

35 C. M. Snapper and W. E. Paul, Interferon-gamma and B cell stimulatory factor-1 reciprocally regulate $I_{g}$ isotype production, Science, 1987, 236, 944-947.

36 A. B. Pavel, J. W. Glickman, J. R. Michels, S. Kim-Schulze, R. L. Miller and E. Guttman-Yassky, Th2/Th1 Cytokine Imbalance Is Associated with Higher COVID-19 Risk Mortality, Front. Genet., 2021, DOI: 10.3389/fgene.2021. 706902.

37 C. W. Tan, W. N. Chia, X. Qin, P. Liu, M. I. C. Chen, C. Tiu, Z. Hu, V. C.-W. Chen, B. E. Young, W. R. Sia, Y.-J. Tan, R. Foo, Y. Yi, D. C. Lye, D. E. Anderson and L.-F. Wang, A SARS-CoV-2 surrogate virus neutralization test based on antibody-mediated blockage of ACE2-spike protein-protein interaction, Nat. Biotechnol., 2020, 38, 1073-1078.

38 A. Manenti, M. Maggetti, E. Casa, D. Martinuzzi, A. Torelli, C. M. Trombetta, S. Marchi and E. Montomoli, Evaluation of SARS-CoV-2 neutralizing antibodies using a CPE-based colorimetric live virus micro-neutralization assay in human serum samples, J. Med. Virol., 2020, 92, 2096-2104.

39 International Clinical Trials Registry Platform. Identifier RPCEC00000338. Phase I, randomized, double-blind and adaptive study to evaluate the safety, reactogenicity and explore the immunogenicity of different formulations of the Prophylactic Vaccine Candidates against SARS-CoV-2, FINLAY- FR-1 and FINLAY- FR-1A (COVID-19). https://rpcec. sld.cu/en/trials/RPCEC00000338-En.

40 S. Perez-Rodriguez, M. C. Rodriguez-Gonzalez, R. Ochoa-Azze, Y. Climent-Ruiz, A. Gonzalez-Delgado, B. Paredes-Moreno, C. Valenzuela-Silva, L. Rodriguez-Noda, R. Perez-Nicado, R. Gonzalez-Mugica, M. Martinez-Perez, B. SanchezRamirez, T. Hernandez-Garcia, A. Diaz-Machado, M. Tamayo-Rodriguez, A. Martin-Trujillo, J. Rubino-Moreno, A. Suarez-Batista, M. Dubed-Echevarria, M. Teresa PerezGuevara, M. Amoroto-Roig, Y. Chappi-Estevez, G. BergadoBaez, F. Pi-Estopinan, G.-W. Chen, Y. Valdes-Balbin, D. Garcia-Rivera and V. Verez-Bencomo, medRxiv, 2021, DOI: $10.1101 / 2021.10 .04 .21264522$. 